\title{
Dementia and COVID-19 Lockdown: More Than a Double Blow for Patients and Caregivers
}

\author{
Raffaella Migliaccio ${ }^{\mathrm{a}, \mathrm{b}, \mathrm{c}, \mathrm{d} *}$ and Arabella Bouzigues ${ }^{\mathrm{a}, \mathrm{d}}$ \\ a INSERM U1127, Institut du cerveau, Sorbonne Université, Hôpital Pitié-Salpêtrière, Paris, France \\ ${ }^{\mathrm{b}}$ Centre de Référence des Démences Rares ou Précoces, Hôpital Pitié-Salpêtrière, Assistance Publique-Hôpitaux \\ de Paris, Paris, France \\ 'Institute of Memory and Alzheimer's Disease, Centre of Excellence of Neurodegenerative Disease, Department \\ of Neurology, Hôpital Pitié-Salpêtrière, Assistance Publique-Hôpitaux de Paris, Paris, France \\ ${ }^{\mathrm{d}}$ FrontLab, Institut du cerveau (ICM)
}

Accepted 13 June 2020

\begin{abstract}
In dementia research and in clinical practice, the COVID-19 pandemic represents an important challenge, not only for neurological staff and researchers, but above all for patients and their caregivers. It is important that the medical staff demonstrate flexibility, open-mindedness, and humanity when following patients and caregivers. It seems inevitable that caregivers will pay the highest price during this crisis.
\end{abstract}

Keywords: Caregiver, collateral damage, COVID-19, dementia, lockdown

\section{INTRODUCTION}

As recently highlighted by a group of dementia experts in the Lancet journal [1], the COVID-19 pandemic joins another pandemic which has been ongoing for many decades; that of dementia. Due to the aging population with which we are faced, dementia is an emerging pandemic [2]. Currently, more than 50 million people have dementia worldwide [3], with the cost of care estimated to reach $\$ 1$ trillion by $2030^{1}$. Dementia is associated with varying degrees of cognitive decline and behavioral changes which have a huge impact on the quality

\footnotetext{
${ }^{*}$ Correspondence to: Raffaella Migliaccio, Institut du cerveau (ICM), Inserm Unit 1127, 47 bd de l'hôpital, 75013, Paris. E-mail: lara.migliaccio@gmail.com.

${ }^{1}$ https://www.alz.co.uk/research/world-report-2015
}

of life of those affected and their relatives. During normal times, patients affected by dementia are very vulnerable people and are hugely dependent on family or professional caregivers in their everyday life. This COVID-19 pandemic worsens their vulnerability directly, because of the virus' morbidity and mortality and, indirectly, because of the lack of social and healthcare support which they depend upon. The double shock of dementia and COVID-19 pandemics has raised major concerns for people with dementia but also their caregivers.

\section{DEMENTIA RESEARCH DURING COVID-19 PANDEMIC LOCKDOWN}

At the moment, we have two emergencies: the first concerns research. As announced by the editors of the Alzheimer's and Dementia journal [4], every effort 
must be made at this time to keep ongoing experimental protocols running, especially those with a therapeutic objective. It takes a very long time to obtain results within clinical research and stopping trials now would be a serious error. The dementia pandemic cannot wait for the COVID-19 pandemic to end for two reasons. Firstly, patients often have to wait a long time before they are included in a clinical trial or research protocol. Secondly, many patients, especially those affected by early dementia, often present rapid cognitive decline. Thus, the present situation represents a "loss of opportunity" for patients. Moreover, for some types of research, those which focus on rare dementias for instance, this will lead to a reduced sample of patients included and have consequences on the quality of the work. As researchers, we believe that the scientific community must establish a real worldwide network of collaborations to find solutions for these issues. The experience of COVID-19 is teaching us that working all together is possible, putting aside one's academic interests because the survival of our society is at stake today. These same attitudes should therefore also be adopted in our fight against dementia.

\section{DEMENTIA CARE DURING COVID-19 PANDEMIC LOCKDOWN}

\section{Response from healthcare professionals}

The second emergency concerns the care provided to dementia patients, in the broadest sense of the term. Amidst the COVID-19 crisis, healthcare professionals have found themselves catapulted into a new reality which has required fast adaptations because of the high risk of contagion in their workplace. Thus, neurologists have been obliged to move from the classic outpatient visit to telemedicine, often in the absence of suitable facilities [5], to cancel all hospitalizations considered as non-urgent, even those which would provide a diagnosis for patients who have been waiting for months, and finally to reconvert from our usual activity to help our colleagues at the forefront of the COVID-19 pandemic. More importantly, during our patients' appointments, we must not only deal with the problems related to dementia, but we must also cope with this new and unexpected scenario and its consequences.

Moreover, it is not just the patient assessment which has been forced to adapt to the new circumstances, but all aspects within healthcare. For example, disinfecting the workplace environment, which can be rather straightforward in a consultation room, becomes more complicated, for example, in places used for diagnostic procedures (biopsies, MRI, etc.). Also, nervous system autopsies can be highly impacted by the virus. Interestingly, to contain the infection risk, specific operating protocols for safe autopsy practice have been issued [6].

The global procedures currently adopted by the entire healthcare world to face this risk of contagion are highly time-consuming. During these times, all the medical workforce, including those within neurology, must demonstrate great flexibility, openmindedness and profound humanity.

\section{Response from dementia patients and caregivers}

Moreover, this crisis is making patients and caregivers even more disoriented than their existing condition already makes them. Many patients live with their partner, who is their primary caregiver. Dementia already isolated them to a certain extent, and with varying degrees, from their family and friends, but now they are physically confined to their home. This situation can come after waiting for months for a suitable therapist for cognitive rehabilitation, for appointments with physicians, and for a place in a dementia care unit which will stimulate patients and give their families a break. Suddenly, it has been announced that all the daily activities which they struggled to set up could no longer take place. This huge modification in everyday life, associated with the inability of dementia patients to manage the present situation, makes these current times hugely demanding for patients and their families. Moreover, caregivers are frustrated by the time and effort which they may have lost, worried that this will setback their loved ones' progress, whilst also living in a very trying context themselves.

The amnestic patients I (R.M.) have been interviewing do not remember the existence of a virus that keeps them confined to their home. The conversation often takes place similarly to this one with Mrs. P:

"Good morning Mrs. P, how do you feel today?"

"Well, doctor, thank you."

"Aren't you rather annoyed by the current situation? By the fact that you can't go out?"

“... what situation? I don't understand.”

"Have you heard of the coronavirus?" 
In a small percentage of cases the word "coronavirus" rapidly evokes a memory, which after a few minutes will probably disappear again. In most cases, patients have confused ideas ranging from "not knowing/not remembering" to "confabulating interpretations". Mrs. P replied to my question saying: "I don't understand"; and after I explained the meaning of my question, she said: "I don't know, I let my husband deal with this, I don't care".

Another patient told me he believed there was a war outside that prevented him from going out. More generally, many of them have difficulties remembering safeguard procedures, such as washing their hands frequently or wearing masks when needed, or understanding the public health information issued to them.

In dementia patients with behavioral problems who struggle to follow the rules, abiding to the regulation of not leaving the house, or avoiding close contact with the people they meet is exceedingly difficult. I recently handled the case of a patient suffering from a frontotemporal dementia and in complete denial of the COVID-19 pandemic. He continued to go out every day: at least three hours in the morning for shopping, and three hours in the afternoon for walking. His wife was panicking and asked me how she could dissuade him from doing so. She had tried to explain the situation to him but the patient had replied: "It is not true! Nothing of this is happening! You just say it because you don't want to let me out of the house!". She tried to convince him to stay at home by organizing interesting activities, but this just caused increased agitation and agressiveness. In order to ensure a rapid control of this situation, I decided to prescribe small doses of antipsychotics. In the meantime, I proposed a compromise; his wife could let him go out, which would also help him learn to respect safeguard procedures (such as wearing a mask when outside, washing his hands often, leaving his shoes outside of the flat, and changing clothes every time he came back from outside), while she waited for the drug to take effect. After starting the treatment, we communicated on the phone every two days. In less than a week, the patient was less agitated and more conciliatory. During our last conversation, the patient had agreed not to go out and spend time at home doing recreational/social activities, like, for example, writing to family and friends to ask for news. For this type of patients, ignoring the warnings and sufficient self-quarantine measures could expose them and their caregivers to higher chances of infection. The neurologist's intervention is therefore key in protecting, not only the patient, but also the caregiver.

\section{Dementia patients in nursing homes}

During the COVID-19 pandemic, individuals with dementia living in nursing homes show several additional problems. Firstly, physical distancing is impossible because of their dependence on healthcare workers. Moreover, as we have observed across the world, personal protective equipment has often been rationed for confirmed or suspected cases of COVID-19, or even unavailable, especially at the beginning of the pandemic [7]. Thus, contagion and fatality rates have been very high in nursing homes. The significant spread of the virus in nursing homes appeared in France as early as the end of February. Some of my (R.M.) patients died very rapidly due to problems that, only today, can be attributed to COVID-19 (acute respiratory syndrome associated with thrombo-embolic phenomena). Due to their vulnerability and this high contagion rate, the number of patients deceased in nursing homes represent a very high proportion in many countries across the world. In the US, for example, the case fatality rate reached 34 among 101 affected residents within a nursing home in Washington state [8].

This has led to a strong fear of contagion among healthcare workers, and has increased patient isolation. I witnessed an example of this during lockdown, when I wished to talk by video call to one of my patients suffering from dementia with Levy bodies, with a mild cognitive deficit, and living in a nursing home. When I finally managed to talk with one of the nurses from the facility, I learned that the patient had got coronavirus SARS-CoV-2. For this reason, the nurse categorically refused to let me speak to him because this implied her entering his room.

In the short term, we must find a way to reduce the spreading of the infection. New care models should be adopted within these communal living environments. This could involve reducing the number of patients per care unit and the number of healthcare professionals per patient, as well as the implementation of technology to maintain contact with the outside world.

In the long term, in view of our knowledge concerning the impacts of environmental, lifestyle, and psycho affective factors on patients with dementia, we should aim to translate this to programs focused on improving their global living conditions. For instance, tailoring the environment to patients in 
order to reduce their stress and anxiety levels by taking into account specific psycho affective and lifestyle aspects.

\section{The place of caregivers and their health}

Several dementia-related societies have given general advice for dementia care giving during the COVID-19 pandemic. $^{2}$ They highlight the importance of, for example, identifying early signs of infection and other diseases in dementia patients, remembering important hygiene practices from one day to the next, thinking ahead and making alternative plans for the person with dementia should adult day care, respite, or other organized events be modified or cancelled in response to COVID-19 or in the situation that the primary caregiver becomes sick. All this advice is centered on the dementia patient. Except for a few scattered sentences, there is no mention of the caregiver's health, and even these are limited to advising on how to prevent becoming overwhelmed by anxiety or panic. Like everyone in this moment, the caregiver has to change their lifestyle, but in this specific case, a dementia caregiver becomes a therapist, a nurse, a rehabilitator, and often without any form of compensation, be it financial or even respite. With all these responsibilities and such immense burden, caregivers are at risk of forgetting about their own health.

There are various measures which can preserve caregivers' health. First of all, caregivers need psychological support, to help them identify and manage their emotions, especially anxiety and anger. Secondly, they need to deal with social isolation by learning to connect with the rest of their family and friends in new ways (frequent phone calls, but also video calls, etc.). It is well known that social isolation among older adults is a "serious public health concern" because of their increased risk of cardiovascular, autoimmune, neuro cognitive, and mental health problems [9]. "Self-isolation will disproportionately affect elderly individuals whose only social contact is out of the home, such as at daycare venues, community centres, and places of worship. Those who do not have close family or friends, and rely

\footnotetext{
${ }^{2}$ For example: Alzheimer Europe at https://www.alzheimereurope.org/Living-with-dementia/COVID-19; Alzheimer Association at https://www.alz.org/help-support/caregiving/coronavirus(covid-19)-tips-for-dementia-care; Alzheimer's Society at https:// www.alzheimers.org.uk/get-support/coronavirus-covid-19; the association for frontotemporal degeneration at https://www. theaftd.org/living-with-ftd/covid-19-and-ftd/
}

on the support of voluntary services or social care, could be placed at additional risk, along with those who are already lonely, isolated, or secluded" [10]. Finally, caregivers' physical health must also be supported. By talking to them, I have come to realize how hard it is. Neurologists and all medical staff must remind caregivers that all their own medical consultations for cancer, cardiologic problems or other serious illnesses should not be delayed as these could become fatal if not addressed in time. In this context of dementia, COVID-19 pandemic lockdown, and social isolation, one of my patients' wife died during her sleep. She had heart problems that needed to be addressed, but as she was at home alone with her husband who suffers from dementia, she was overwhelmed and didn't take appropriate measures for her own health. French authorities ${ }^{3}$ alerted about the risk of very serious collateral damage, because "in the context of COVID-19 pandemic, patients no longer consult their medical doctors for other reasons than for COVID-19", and they strongly suggest that the fear of contamination by the virus linked with COVID-19 should not prevent recourse to treatment, in particular for patients suffering from pathologies requiring regular monitoring or for children.

\section{CONCLUSIONS}

At the end of this pandemic, the damage for dementia patients and their primary caregivers could be immense, not only because of the number of deaths related to the virus (especially those in nursing homes), but also considering the "collateral" damage due to a faster evolution of demented patients who are less supported and stimulated and who are not seeking treatment for other physical and psychological problems which they or their caregiver may require. This collateral damage can be prevented to a certain extent, if the appropriate advice and help is given to individuals who face the difficulties of dealing with dementia in this unprecedented time.

\section{ACKNOWLEDGMENTS}

Raffaella Migliaccio thanks all her colleagues (especially Isabelle Le Ber and Roberto Arienzo),

\footnotetext{
${ }^{3}$ Agence régional de Santé at https://www.iledefrance.ars. sante.fr/continuite-des-soins-pendant-la-periode-epidemiquecovid-19; Haute Autorité de Santé at https://www.has-sante. fr/jcms/p_3168634/fr/assurer-la-continuite-de-la-prise-en-chargedes-personnes-atteintes-de-maladies-chroniques-somatiquespendant-la-periode-de-confinement-en-ville
} 
her patients and their courageous caregivers. Raffaella Migliaccio is supported by "France Alzheimer" and "Philippe Chatrier" Foundations, by "Fondation recherche Alzheimer" and by "Rosita Gomez association". Arabella Bouzigues thanks "Fondation Vaincre Alzheimer".

\section{CONFLICT OF INTEREST}

The authors have no conflict of interest to report.

\section{REFERENCES}

[1] Wang H, Li T, Barbarino P, Gauthier S, Brodaty H, Molinuevo JL, Xie H, Sun Y, Yu E, Tang Y, Weidner W, Yu X (2020) Dementia care during COVID-19. Lancet 395, 1190-1191.

[2] Fox NC, Petersen RC (2013) The G8 Dementia Research Summit—a starter for eight? Lancet 382, 1968-1969.

[3] Alzheimer's Disease International (2019) World Alzheimer Report 2019. Attitudes to dementia. Alzheimer's Disease International, London, UK.

[4] (2020) Alzheimer's disease research enterprise in the era of COVID-19/SARS-CoV-2. Alzheimers Dement 16, 587-588.
[5] Klein BC, Busis NA (2020) COVID-19 is catalyzing the adoption of teleneurology. Neurology 94, 903-904.

[6] Aquila I, Sacco MA, Abenavoli L, Malara N, Arena V, Grassi S, Ausania F, Boccuto L, Ricci C, Gratteri S, Oliva A, Ricci P (2020) SARS-CoV-2 pandemic: review of the literature and proposal for safe autopsy practice. Arch Pathol Lab Med, doi: 10.5858/arpa.2020-0165-SA

[7] Ranney ML, Griffeth V, Jha AK (2020) Critical supply shortages - The need for ventilators and personal protective equipment during the Covid-19 pandemic. $N$ Engl $J$ Med 382, e41.

[8] McMichael TM, Currie DW, Clark S, Pogosjans S, Kay M, Schwartz NG, Lewis J, Baer A, Kawakami V, Lukoff MD, Ferro J, Brostrom-Smith C, Rea TD, Sayre MR, Riedo FX, Russell D, Hiatt B, Montgomery P, Rao AK, Chow EJ, Tobolowsky F, Hughes MJ, Bardossy AC, Oakley LP, Jacobs JR, Stone ND, Reddy SC, Jernigan JA, Honein MA, Clark TA, Duchin JS (2020) Epidemiology of Covid-19 in a long-term care facility in King County, Washington. $N$ Engl J Med 382, 2005-2011.

[9] Gerst-Emerson K, Jayawardhana J (2015) Loneliness as a public health issue: the impact of loneliness on health care utilization among older adults. Am J Public Health 105, 1013-1019.

[10] Armitage R, Nellums LB (2020) COVID-19 and the consequences of isolating the elderly. Lancet Public Health $\mathbf{5}$, e256. 